\title{
Inglés en tiempos de CLL: propuesta para una nueva metodología para las clases de inglés
}

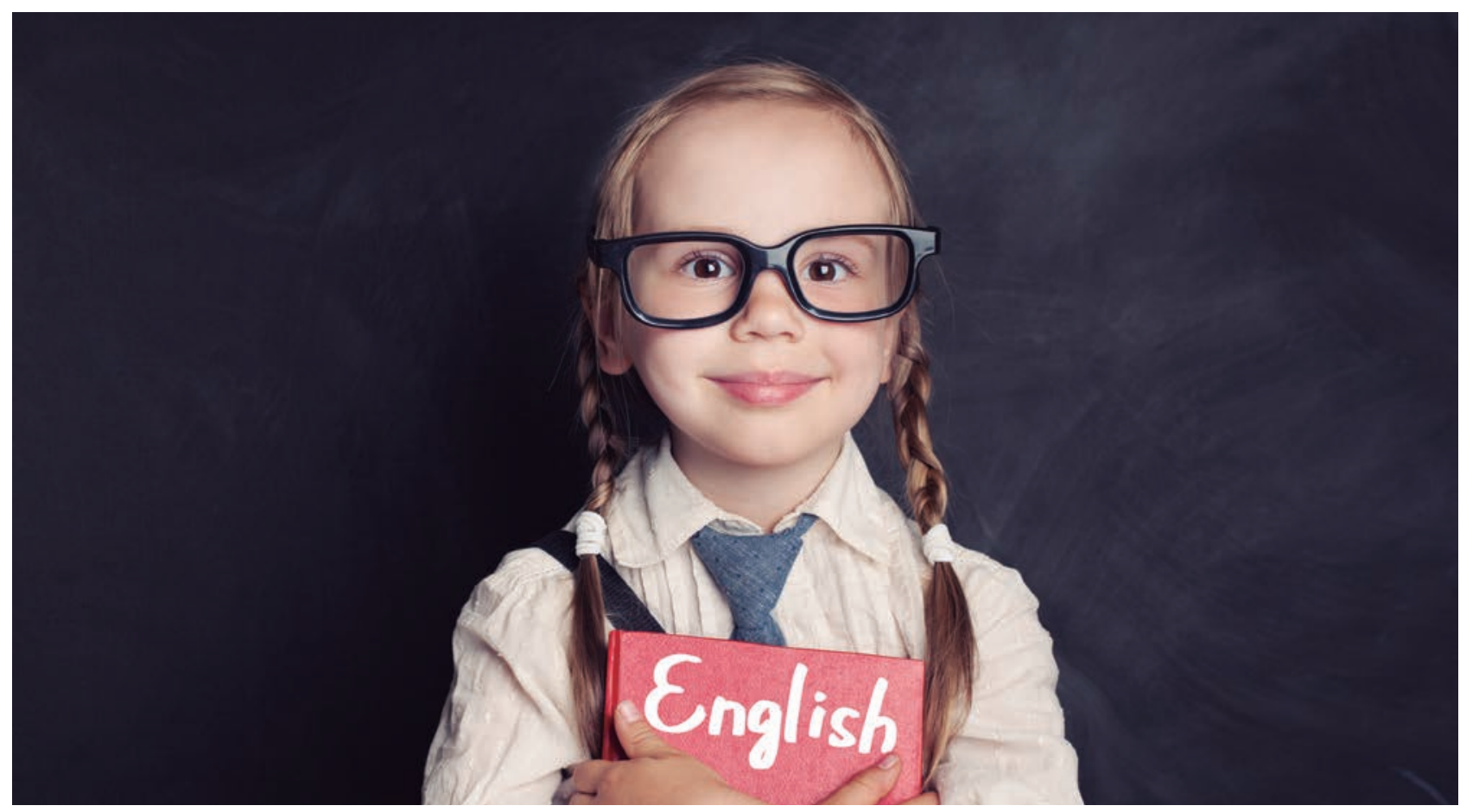

"Hemos encontrado el libro de texto ideal este año. No hay nada que mejorar". Así me contestó una alumna del máster, profesora de primaria en un centro bilingüe, a la que le acababa de proponer que llevara a cabo un proyecto de trabajo con textos en su clase de

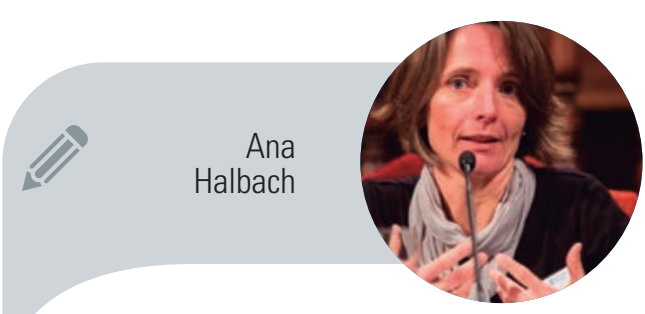

Universidad de Alcalá de Henares ana halbach@uah.es web: https://inglesenbilingue.blogspot.com/ (2) @Ana19445340 inglés. Un par de meses más tarde, hecha la experiencia, no querían saber nada del libro de texto ni ella ni sus alumnos. CLIL pide a gritos un cambio metodológico — también en las clases de inglés-. 
Hace ya algunos años que vivimos "en tiempos de CLIL". En prácticamente todos los rincones de España, y en muchos países de nuestro entorno, la lengua extranjera, mayoritariamente el inglés, se ha convertido en la lengua de las clases de Ciencias Sociales, Biología o de la tutoría, entre otros. En los centros educativos se habla inglés y se utiliza como herramienta para el aprendizaje. En esto consiste, a ojos de la mayoría, la integración que recoge el término CLIL (Content and Language Integrated Learning) o AICLE en sus siglas en español (aprendizaje integrado de contenidos y lenguas extranjera). Sin embargo, hay otro elemento en nuestro currículo que es fundamental en esta ecuación y que muchas veces se queda al margen de la misma: la asignatura de Lengua Extranjera. Parecería que allí no hay nada que integrar, pero ¿y si la integración se produjera entre las clases de contenido impartidas en inglés y las clases de inglés? Porque, teniendo un coche híbrido con dos formas de propulsión, ¿a alguien se le ocurriría utilizarlo solamente con gasolina?

\section{Una nueva metodología para las clases de inglés}

Sin embargo, para que podamos utilizar estas dos formas de propulsión y así llegar más lejos en nuestro aprendizaje, es necesario adaptar tanto la metodología utilizada en las clases de inglés como la forma de planificar nuestra enseñanza, adoptando un enfoque más global de la misma. En los proyectos de enseñanza bilingüe el papel de la lengua extranjera cambia, y en vez de ser un objeto de estudio se convierte (ipor fin!) en herramienta de comunicación, y lo hace de manera natural porque de hecho los alumnos necesitan esta lengua para aprender. Ocurre, sin embargo, que muchas veces el enfoque que se le da a la enseñanza del inglés, incluso en estos contextos que propician la comunicación, sigue estando muy centrado en el estudio de la gramática y el vocabulario, y de esta manera no funciona adecuadamente como motor

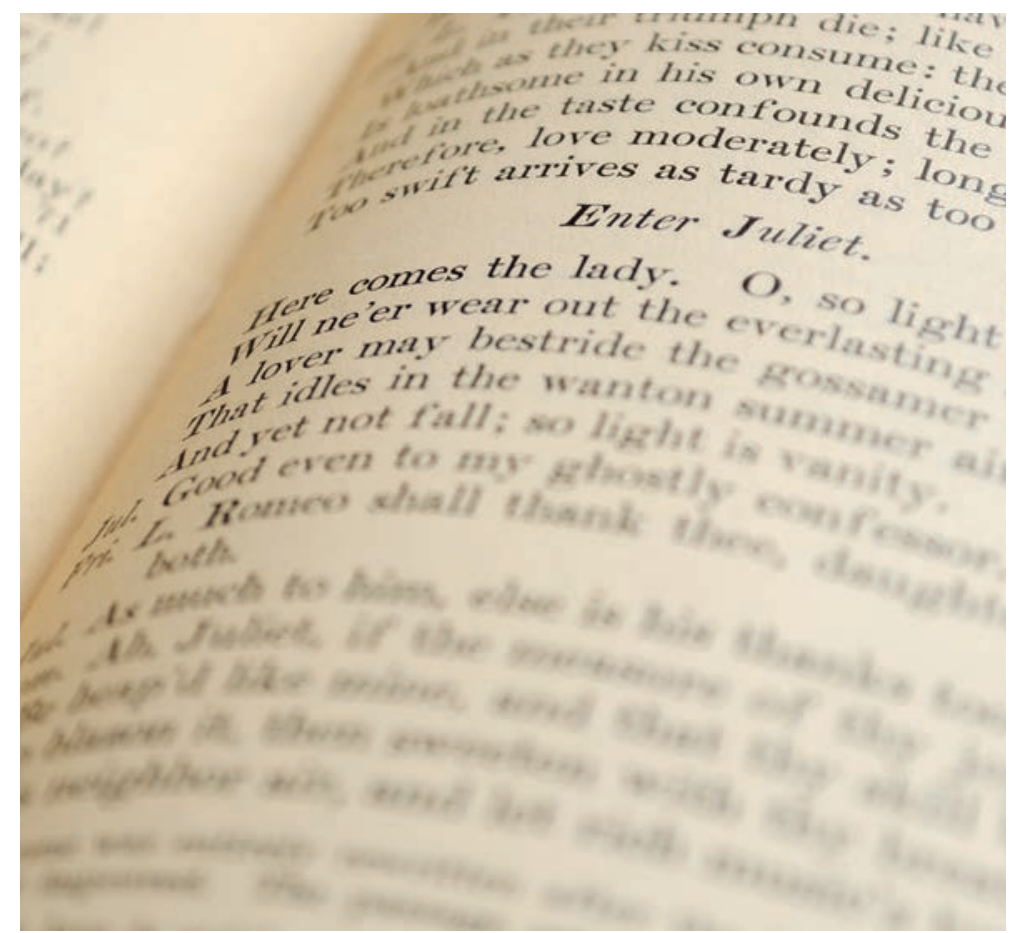

de propulsión de este coche híbrido que nos hemos comprado.

Hace algunos años, en una visita a un centro de primaria con proyecto bilingüe, pregunté a los profesores encargados de dar la asignatura de inglés si habían notado un cambio en los alumnos del proyecto, a lo cual, con amplia sonrisa de satisfacción, me contestaron "sí, claro, ahora en vez de dar un libro de texto al año damos un libro y medio". Este comentario refleja la primera característica, que no la más importante, de lo que cambia en el trabajo en las clases de lengua extranjera en un proyecto bilingüe: Ios alumnos aprenden con mayor rapidez. Sin embargo, hay otras características que seguramente tendrán un mayor impacto sobre el cambio metodológico que necesitamos.

En primer lugar, los alumnos están acostumbrados a utilizar la lengua para comunicarse y aprender en las clases de contenido impartidas en inglés. Esto quiere decir que no solamente están dispuestos a utilizarla también en sus clases de inglés, sino que insistir en un enfoque que se centra en el estudio de la gramática y el vocabulario resulta poco motivador y menos relevante para este tipo de alumnado. Pero no solamente pueden y quieren utilizar la lengua como herramienta de comunicación -lo que, de acuerdo con las teorías de adquisición de segundas lenguas, llevará a un mejor aprendizaje de las mis- 


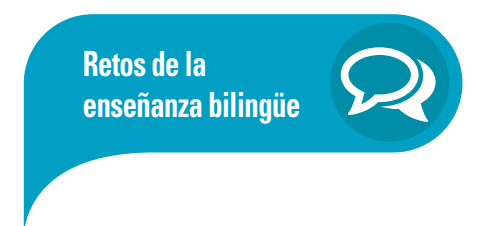

ACTIVIDADES DE AULA

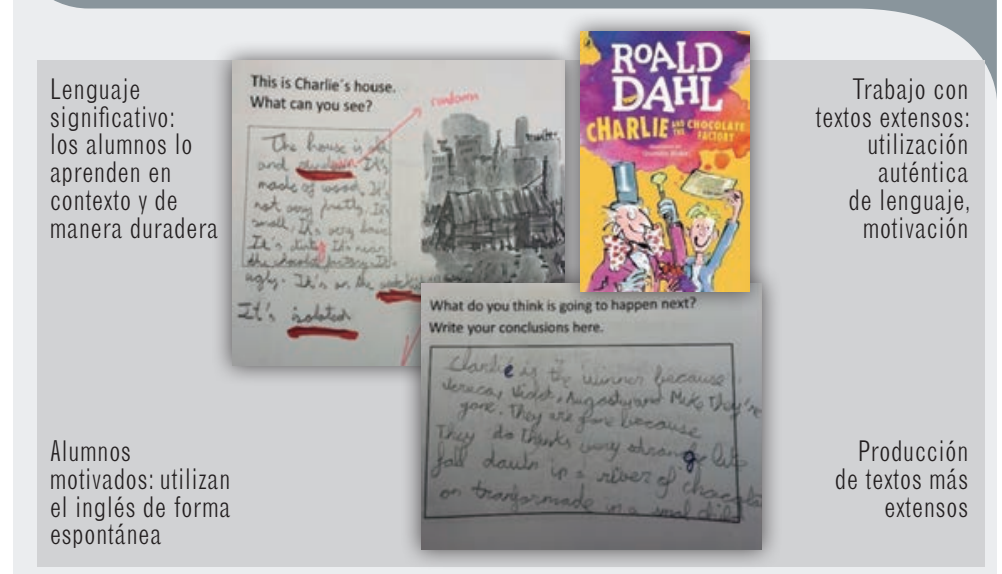

$y \quad$ Lectura de un texto extenso y motivador

y Trabajo de comprensión y de desarrollo de las estrategias de lectura

y Utilización de extractos del texto como modelos de tipos de texto

y Identificación de alguna forma linguística significativa dentro del texto

$\checkmark \quad$ Propuesta de producción libre modelada en el extracto del texto

En este caso el soporte es un texto escrito, pero el mismo esquema se puede utilizar para los textos orales. papel fundamental, ya que es el lugar en el que se puede hacer este trabajo de desarrollo de las destrezas comunicativas y de las estrategias. De la misma manera, en la clase de inglés se puede preparar el terreno para lo que ocurre en las clases de contenidos, de manera que, si en la clase de Ciencias Naturales vamos a comparar los planetas, previamente, en clase de inglés, podemos haber trabajado los comparativos. Y haciéndolo no solamente contribuimos a que los alumnos se expresen con corrección en su clase de Ciencias Naturales, sino también a que la lengua pueda servir para aprender, ya que verbalizando lo que sé es como aprendo.

Este tipo de trabajo en el que la asignatura de inglés prepara el terreno a la asignatura de contenido requiere de una planificación previa y, en ocasiones, si las asignaturas las imparten profesores diferentes, de una importante labor de coordinación. Para poder apoyar la docencia en las asignaturas de contenido desde el punto de vista comunicativo, lingüístico y estratégico, el profesor de inglés debe saber de antemano qué es lo que requiere la asignatura de contenido en este sentido. Pero no por ello las clases de inglés se convierten en una extensión de las clases de contenido; esa misma preparación se puede (y creo que se debe) hacer en el contexto en el que se realiza normalmente la comunicación: el texto hablado y escrito.

\section{Trabajar con textos}

Dice Peter Mickan (2017) que la mayor parte de nuestra comunicación se realiza en forma de textos - entendidos como cualquier producción lingüística que vaya más allá de un sonido o una palabra-y que resulta curioso ver cómo en las clases de lengua extranjera aislamos los elementos de la comunicación, principalmente las estructuras gramaticales y el vocabulario, los enseñamos y esperamos que los alumnos, por ciencia infusa, sean capaces de volver a utilizarlos como parte de un texto. ¿No sería mucho más lógico que el trabajo se realizara directamente en la situación comunicativa en la que se enmarca de forma natural, es decir, un texto 
hablado o escrito? De esta manera, el vocabulario o las estructuras gramaticales que se trabajan son más significativas ya que existe un contexto y una necesidad real de utilizarlos y encima las destrezas comunicativas se desarrollan de manera auténtica leyendo o escuchando un texto, hablando sobre él, escribiendo una respuesta o un texto similar, etc.

Así, podemos trabajar diferentes tipos de texto con mayor o menor complejidad y ayudar a que los alumnos perciban y entiendan, no solamente cómo funcionan el vocabulario y las estructuras gramaticales de este texto, sino cómo se estructuran los textos, cómo se ordenan las ideas en una explicación coherente o cómo se consigue que una descripción sea sugerente. Esto supone desarrollar la competencia comunicativa y desarrollar las habilidades necesarias para que los alumnos puedan enfrentarse con éxito al aprendizaje de contenidos en inglés.

De esta manera, podemos trabajar la descripción de la casa en la que vivimos utilizando como punto de partida el cuento There is a Bird on your Head de Mo Willems o ayudar a los alumnos a crear sus propios trabalenguas utilizando algunos de los más conocidos como modelo. En el primer caso, los alumnos aprenden las estructuras "There is / there are" en el contexto del cuento y luego las transfieren a la actividad prevista en el currículo (describir la casa en la que vives), mientras que la segunda propuesta se centra en la pronunciación y en la identificación de sonidos similares en diferentes palabras, además de fomentar la creatividad de los alumnos. De igual manera, podemos trabajar la descripción de monstruos a partir del modelo encontrado en The Gruffalo de Julie Donaldson o, en un nivel superior, trabajar la descripción de una casa partiendo de la descripción que se encuentra en Charlie and the Chocola-

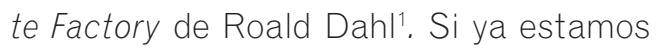
pensando en alumnos de los últimos cur-

$1 \quad$ Agradezco a las profesoras Cristina Díaz, Eva Jechimer y Sandra Torralvo estas excelentes sugerencias.

Para muchos padres la enseñanza bilingüe está repleta de interrogantes: ¿aprenderá mi hijo los mismos contenidos que si aprendiera en un centro no bilingïe? ¿Se resentirá su nivel de lengua materna con tantas horas de exposición al inglés? ¿Cómo puedo apoyarle si no sé inglés? Cada una de estas preguntas necesitaría una reflexión más amplia, pero aquí van unas pocas ideas que pueden, al menos, empezar a resolverlas.

CLIL no consiste solamente en cambiar el idioma de comunicación en el aula, sino que implica un cambio de metodología, ya que los profesores son conscientes de la dificultad añadida de adquirir unos conocimientos y desarrollar unas competencias mediante una lengua que no es la materna. Esto no cambia el contenido de las asignaturas que es exactamente igual en los centros bilingïes que en los no bilingïes. Prueba de ello son los resultados de las pruebas de evaluación, en las que muchas veces los alumnos de centros bilingïes obtienen mejores resultados a pesar de que se examinan en castellano.

Esto demuestra también que el desarrollo de la lengua materna no se ve perjudicado por la enseñanza bilingïe. De hecho, los estudios demuestran que los niños que crecen expuestos a más de una lengua suelen desarrollar una conciencia linguística superior a los niños monolingües. Eso sí, todo depende de la calidad y la cantidad del input al que están expuestos, por lo que es aquí donde los padres que no sepan inglés pueden apoyar a sus hijos: leyendo cuentos en español, hablando con los niños, escuchando sus relatos, etc.

Para más información práctica véase: M. Van Wechem \& A. Halbach (2014), Don’t worry mum and dad. I will speak English. Disponible en https://www.britishcouncilschool.es/ sites/default/files/british_council_guia_bilinguismo.pdf

Tabla 1. Comparación entre metodología tradicional y metodología basada en textos

Metodología tradicional

Textos escritos para ilustrar una estructura gramatical

Trabaja las cuatro destrezas comunicativas

Trabajo a nivel oracional

Lengua como objeto de estudio

Trabaja las estructuras de manera aislada

El objetivo de la unidad es dominar una estructura gramatical

La producción oral o escrita viene al final de la unidad y no existe andamiaje para ella Programa y secuenciación propios

sos de enseñanza primaria, podemos centrarnos en la descripción de personajes a partir de las descripciones que podemos encontrar en el primer volumen de Harry
Metodología basada en textos

Textos auténticos

Integración de las cuatro destrezas: se leen y escuchan los textos que se trabajan; se habla de los textos, se analizan, se responde a ellos y se produce un texto similar (oral 0 escrito) Trabajo a nivel de texto

Lengua como modo de expresión de una intención comunicativa

Estudio de la lengua en el contexto de un texto (oral o escrito): aprendizaje significativo y duradero El objetivo de la unidad es producir un determinado tipo de texto (oral o escrito) con unas características específicas

Toda la unidad va encaminada a hacer posible la producción oral o escrita

Puede apoyar las necesidades lingüísticas de los alumnos en las asignaturas de contenido 


\section{IIII.) ÂQORA DE PROFESORES}

Para apoyar la labor de los profesores de inglés en el cambio metodológico que propongo, he creado un blog abierto al público:

A. НАLваCH (2018), Inglés en enseñanza bilingüe [Blog], Recuperado de https://inglesenbilingue.blogspot.com/. En él intento combinar las reflexiones metodológicas con propuestas concretas de actividades, en principio centradas en la etapa de secundaria, pero fácilmente adaptables a las características del alumnado de Educación Primaria.
Potter. En todos los casos existe una producción final por parte del alumno que parte de un modelo propuesto en clase. Sin embargo, este texto modelo tiene una función más allá de servir de modelo, ya que constituye un texto que se puede leer o escuchar, disfrutar, entender, del que se puede hablar, etc. Se desarrollan las destrezas comunicativas y al mismo tiempo se introducen los elementos lingüísticos de forma significativa, como parte de un hecho comunicativo y no como objeto de estudio. Esto cambia radicalmente la motivación de los alumnos, su percepción de la importancia del trabajo que están haciendo, su aprendizaje a largo plazo y su disfrute con los textos. De la misma manera, al ser un aprendizaje significativo y contextualizado, a los alumnos le costará mucho menos transferir lo aprendido en la clase de inglés a las asignaturas no lingüísticas.

\section{El efecto de la enseñanza centrada en torno a los textos}

La experiencia de los que han puesto en práctica este acercamiento a la enseñanza del inglés demuestra que, también para los profesores, esta forma de trabajar resulta más motivadora además de satisfactoria, al ver los resultados de aprendizaje de los alumnos. Esto no quita que suponga, también, mucho trabajo de preparación y una cierta sensación de salto al vacío: donde antes teníamos un programa bien organizado en torno a unas estructuras gramaticales con un libro de texto que nos quitaba la responsabilidad de planificar, ahora tenemos unos objetivos más globales - por ejemplo, describir un monstruo - que implican la utilización de cierto vocabulario y ciertas estructuras pero que van mucho más allá de dominar estos elementos lingüísticos. Dejamos la seguridad de lo conocido, que no por ello efectivo, porque ¿cuántos de nosotros no hemos hecho la experiencia de que por mucho que expliquemos cómo se forma un comparativo en inglés o como se forman las oraciones condicionales los alumnos siguen cometiendo errores? A cambio, nos lanzamos a una aventura que nos promete unos resultados mejores y mayor eficacia en el aprendizaje. Pero, ¿acaso alguno de nosotros hace 15 años, coincidiendo con la implantación de los primeros programas CLIL, imaginaba que los coches tendrían dos formas de propulsión? Aprovechemos que CLIL ha supuesto un cambio metodológico además de contribuir a aumentar de forma significativa el nivel de inglés de nuestros alumnos y descubramos nuevas formas más efectivas de enseñar y aprender inglés. Al fin y al cabo, estamos en tiempos de CLIL •

\section{(CA DRRA SABER MÁS}

HALBACH, A. (2018). A literacy approach to language teaching: a proposal for FL teaching in CLIL contexts. PULSO. Revista de educación, (41), 205-223.

Mickan, P. (2017). Text-based research and teaching from a social semiotic perspective: Transformative research and pedagogy. En P. Mickan \& E. Lopez (eds.), Text-based research and teaching (pp. 15-35). London: Routledge.

\section{HEMOS HABLADO DE}

\section{Enseñanza del inglés como lengua extranjera; Content and Language Integrated Learning (CLIL); tipos de texto; destrezas comunicativas; aprendizaje significativo.}

Este artículo fue solicitado por PADRES Y MAESTROS en diciembre de 2018, revisado y aceptado en marzo de 2019. 